\title{
EDITORIAL
}

\section{The red wolf remains a wily foe}

T he latest report that belimumab, a human monoclonal antibody targeting BAFF (B-cell activating factor, also known as TNF ligand superfamily member $13 \mathrm{~B}$ or BLyS), was not better than placebo in controlling signs and symptoms of systemic lupus erythematosus (SLE) after 76 weeks re-emphasizes the difficulty of developing new drugs for this disease. Previous positive results after 1 year of therapy with belimumab in the BLISS-52 and BLISS-76 trials had been the only positive outcome in recent SLE trials, and had served to stimulate a belief that development of new therapies was not only possible, but also likely. However, the new results reported in a press release not only question the impact of belimumab, but also raise important issues about the underlying assumptions of SLE clinical trials. Is our understanding of SLE immunopathogenesis correct? Do we actually understand the basis of all clinical manifestations of SLE sufficiently to expect positive results from highly targeted therapies? Are the clinical trials designed appropriately? Is patient heterogeneity too great to see a therapeutic impact? Are we able to identify patients with lupus with a sufficiently high probability of responding to warrant further clinical trials? All of these issues, and many others, must be reconsidered before more money, effort and the good will of more patients who participate in trials are squandered in fruitless pursuits. To quote the Major League Baseball player Yogi Berra, "The future ain't what it used to be."

It is often stated that no new therapies have been approved for SLE in the past half century, and this is certainly correct; however, this does not mean that the care of patients with SLE has not improved dramatically. SLE has been transformed from a rapidly fatal disease into a more chronic and manageable one, and patients with this disease now have an improved life expectancy and can lead reasonably normal lives. This, however, does not mean that current therapies are ideal, far from it. The current combinations of immunosuppressive medications are associated with unwanted and serious adverse events, and largely work by dampening signs and symptoms, but are rarely curative. Moreover, as survival has increased, there has been a growing awareness of co-morbidities, such as cardiovascular and infectious diseases, that provide new challenges for physicians and patients alike. It is safe to conclude that current therapy of SLE is far from optimum. Furthermore, the standard immunosuppressive agents most commonly used in SLE, including cyclophosphamide, mycophenylate and azathioprine, have not been approved by the FDA for use in SLE. This adds to the difficulty in designing new trials to test agents in SLE and to the frustration of rheumatologists, as the obvious comparators have not been approved. Interestingly, antimalarial agents have been approved for use in SLE, but so far have not been thoroughly tested against new biologic drugs, probably because of their perceived lower efficacy. To quote Berra again, "In theory there is no difference between theory and practice, in practice there is."

Large randomized controlled studies have examined the impact of targeting $\mathrm{T}$ cells with abatacept and B cells with rituximab (EXPLORER and LUNAR trials) in patients with SLE. Even though an abundance of preclinical work had clearly demonstrated a role of T cells and B cells in SLE, these trials failed to achieve their primary endpoints. There has been considerable discussion in the community about why these trials were unsuccessful.

The first set of issues focuses on the agents themselves. Did they sufficiently prevent activation of or delete the cells relevant to disease pathogenesis? Since there is no way to measure the impact of these agents on the putative specific target cell population, there is no means to answer this question. An effort is being made to develop the appropriate biomarkers during the trials, and not before, and, therefore, it is difficult to know whether an appropriate pharmacodynamic endpoint had been achieved in these trials. Some have even questioned whether the immune system is involved in disease pathogenesis in the patients with long standing SLE who are entered into these trials. Since autologous stem cell transplantation (ASCT) and high-dose chemotherapy (HDCT) appear to be effective in patients with a similar or even more advanced stage of disease, it seems reasonable to conclude that the immune system is involved even in late-stage SLE, although it must be admitted that appropriately controlled trials of ASCT and HDCT have not been carried out to date in SLE. However, considering the failed trials of abatacept and rituximab on the one hand, and the apparently positive effects of ASCT along with classic nonspecific immunosuppressive agents on the other, the question as to whether simultaneous suppression of B-cell and T-cell activity might not be necessary for a successful outcome in SLE is raised. As Berra said, "You can observe a lot by watching."

The second set of concerns relates to the design of the clinical trials, the subjects enrolled, the co-therapies employed and the outcome measures used. All of these are legitimate concerns and could have contributed to the negative results. One additional concern relates to the design of the clinical development programs. Traditionally, an agent is examined in a series of early phase II trials to determine the most effective dosage schedule, perhaps to define appropriate outcomes measures, sample sizes and even to determine subsets

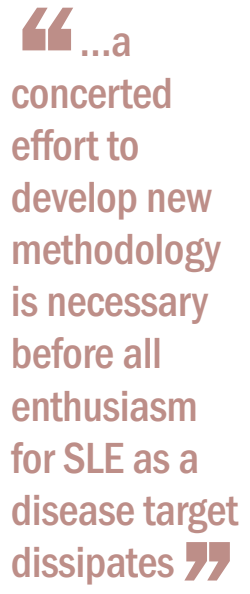

Peter E. Lipsky is the Editor-in-Chief of Nature Reviews Rheumatology. Thomas Dörner is a Professor of

Rheumatology and Clinical Immunology, Department of Medicine,

Rheumatology and Clinical Immunology, Charité

Universitätsmedizin Berlin and Deutsches Rheumaforschungszentrum, Berlin, Germany.

Competing interests

Thomas Dörner

declares associations with the following companies/

organizations: DFG/BMBF,

DRFZ/Senate of Berlin, Immunogenetics Inc., Roche and UCB. See the article online for full details of the relationships. P. E. Lipsky declares no competing interests. 
of patients who might respond favorably, before large definitive studies are carried out. The early trials carried out in phase II are considered to be hypothesis generating and the larger definitive trials in phase III to be hypothesis testing. With rituximab and abatacept, little was done to generate testable hypotheses and approaches that could test them reliably in phase III. One important issue relates to the application of dosages and dosing regimens used in rheumatoid arthritis trials directly into definitive studies of SLE patients, with no proper dose-ranging studies. Consequently, it is not known whether alternative doses or dosage schedules might be more effective. This could have contributed to the negative results. As Berra noted, "If you don't know where you are going, you might wind up someplace else."

A third area of challenge is related to the unique nature of SLE as a waxing and waning disease, associated with the lack of a clear internationally-accepted standard of care. This has implications on the outcome of placebo groups and on the effect size of the putative active compound. There has been a highly variable 'placebo' response in trials from different regions of the world, in some cases approaching 50\%. This has mandated a need for very large trials in order to detect a relatively small effect size on top of a large and often unpredictable placebo response. Notably, the challenge of dealing with a heterogeneous disease with a waxing and waning course have been addressed in multiple sclerosis by delineating subsets of disease with different likelihoods of response, and also developing accepted outcome measures. Perhaps there are lessons here for SLE clinical trials, too. As Berra said, "When you come to a fork in the road, take it!"

The developers of belimumab did extensive phase II testing and determined a subpopulation with a greater likelihood of responding, obtained sufficient data to carry out a power calculation, and identified a composite set of outcome measures that permitted the agent to be distinguished from placebo. Application of the lessons learned in phase II trials to two very large phase III trials (BLISS-52 and BLISS-76) generated positive results at 52 weeks. The entire SLE community was excited about these results because they demonstrated that a set of empirically-defined clinical trial design principles could be applied that permitted the discrimination of a potential SLE therapeutic from placebo. This appeared to provide a pathway that demonstrated the success of belimumab, but also might permit other agents to be appropriately tested. However, SLE is a tough nut to crack, and continuation of one of the trials (BLISS-76) for another 6 months resulted in a loss of the difference between placebo and the putative active agent. To quote Berra, "This is like déjà vu all over again."

It is too early to interpret this result, especially because the data are not yet in the public domain, but the pessimistic view is that even if approved, an agent with a modest effect that is lost after the first year might not have too much utility in a chronic autoimmune disease such as SLE. Moreover, the pathway blocked by belimumab might not be essential for the progression of SLE pathogenesis in the subset of SLE patients enrolled in the trial. More data will be necessary to address these issues.

Besides the impact on belimumab development, the effect of these results on drug development in SLE in general could be negative. There was transient enthusiasm when the initial results of belimumab were positive because the approach seemed to provide a clinical development pathway that might be fruitful. Now, that pathway is in question. One hopes that companies don't leave the field, thinking that SLE is too vexing a disease. There are many interesting agents in development for SLE, including agents that target CD22, interleukin-6, CD154, interferon- $\alpha$, interleukin-21 and others, and one hopes that a pathway forward is found. The sage advice of Berra that "It ain't over 'till it's over" might be useful here. However, it is clear that the SLE community must learn from the unsuccessful trials and, working together, needs to find clinical trial designs and outcome measures that will make it possible to test potential drugs for SLE efficiently and accurately. Repeating failed approaches is unlikely to yield better results. As Berra noted, "If you can't imitate him, don't copy him."

It is clearly a troubling time in the development of new drugs for SLE. There are many outstanding ideas, but no pathway forward that has been demonstrated to be capable of yielding a successful outcome. In this situation, a concerted effort to develop new methodology is necessary before all enthusiasm for SLE as a disease target dissipates. A dialogue between all stakeholders is urgently needed and it is essential for all participants to listen, learn and work together to develop new approaches. Perhaps we can begin by following the advice of Berra again, who noted, "I never said most of the things I said."

doi:10.1038/nrrheum.2010.83 